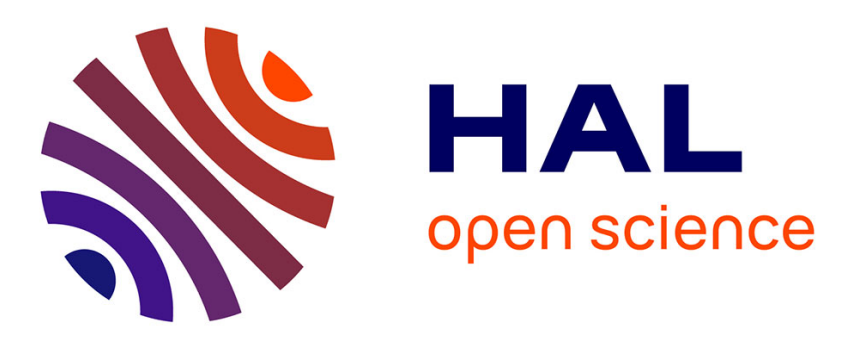

\title{
Identification de sources électromagnétiques multipolaires par filtrage spatial: simplification du contour des capteurs
}

Benjamin Vincent, Olivier Chadebec, Jean-Luc Schanen, Kévin Berger, Laurent Krähenbühl, Ronan Perrussel

\section{To cite this version:}

Benjamin Vincent, Olivier Chadebec, Jean-Luc Schanen, Kévin Berger, Laurent Krähenbühl, et al.. Identification de sources électromagnétiques multipolaires par filtrage spatial: simplification du contour des capteurs. European Journal of Electrical Engineering, 2012, 15 (6), pp.613-632. 10.3166/EJEE.15.613-632 . hal-00639598

\section{HAL Id: hal-00639598 https://hal.science/hal-00639598}

Submitted on 6 Jul 2017

HAL is a multi-disciplinary open access archive for the deposit and dissemination of scientific research documents, whether they are published or not. The documents may come from teaching and research institutions in France or abroad, or from public or private research centers.
L'archive ouverte pluridisciplinaire HAL, est destinée au dépôt et à la diffusion de documents scientifiques de niveau recherche, publiés ou non, émanant des établissements d'enseignement et de recherche français ou étrangers, des laboratoires publics ou privés. 


\title{
Identification de sources électromagné- tiques multipolaires par filtrage spatial : simplification du contour des capteurs
}

\author{
Benjamin Vincent*_ Olivier Chadebec* — Jean-Luc Schanen*- \\ Kévin Berger**_ Laurent Krähenbühl*** _ Ronan \\ Perrussel $* * * *$ \\ * G2Elab, CNRS UMR5269, Université de Grenoble, \\ ENSE $^{3}$, BP 46, 38000 Grenoble, France \\ \{benjamin.vincent, olivier.chadebec, jean-luc.schanen\}@g2elab.grenoble-inp.fr \\ ** Laboratoire GREEN -UHP, Faculté des Sciences et Technologies - BP 70239 \\ 54506 Vandoeuvre lès Nancy Cedex, France \\ kevin.berger@green.uhp-nancy.fr \\ *** Laboratoire Ampère CNRS UMR5005, Université de Lyon, \\ Ecole centrale de Lyon, 69134 Ecully Cedex, France \\ laurent.krahenbuhl@ec-lyon.fr \\ **** LAPLACE, CNRS UMR5213, Université de Toulouse, INPT, ENSEEIHT, F- \\ 31071 Toulouse, France \\ perrussel@laplace.univ-tlse.fr
}

\begin{abstract}
RÉSUMÉ. De nouveaux capteurs d'induction magnétique en champ proche, dédiés aux études de Compatibilité ElectroMagnétique (C.E.M.), sont proposés sur le principe des spires de la norme CISPR16-1. La forme des bobines proposées leur permet de n'être sensibles qu'à certaines composantes multipolaires du champ, ce qui réalise un filtrage spatial. L'exposé fait suite à des travaux antérieurs et se concentre exclusivement sur la méthode de simplification des contours de détection. A l'aide de rotations du repère de référence, il s'agit de simplifier la géométrie des bobines du capteur proposé précédemment. Après un exposé des outils utilisés, la méthode de synthèse de ces nouveaux contours est décrite.

ABSTRACT. New sensors of magnetic induction in near field, dedicated to studies of ElectroMagnetic Compatibility (E.M.C.), are proposed according to the principle of the Standard CISPR16-1 coils. The new coil shape allows the sensors to be sensitive to only some specific components of the multipolar expansion, which is similar to a spatial filtering. Our proposition follows previous works and just focuses on the concept of shape coils simplification. By means of rotations of the reference frame, our aim is to simplify the geometry of the sensor coils introduced before. After a description of the tools required, the design method is described.
\end{abstract}

Revue. Volume $\mathrm{X}-\mathrm{n}^{\circ} \mathrm{x} /$ année, pages 1 à $\mathrm{X}$ 
2 Revue. Volume $\mathrm{X}-\mathrm{n}^{\circ} \mathrm{x} / \mathrm{année}$

MOTS-CLÉS : capteurs électromagnétiques bobinés, mesures en champ proche, développement multipolaire, identification de sources électromagnétiques, C.E.M., harmoniques sphériques réelles, rotation.

KEYWORDS: coils electromagnetic sensors, near field measurements, multipolar expansion, electromagnetic sources characterization, E.M.C., real spherical harmonics, rotation. 


\section{Introduction}

La connaissance des champs électromagnétiques émis par les systèmes d'électronique de puissance permet l'étude de la Compatibilité ElectroMagnétique (C.E.M.) d'ensembles complexes, mettant notamment en jeu plusieurs convertisseurs. Pour ces structures complexes, la modélisation fine de l'ensemble des sources du dispositif reste difficile à mettre en œuvre [AIM09]. Afin de surmonter cette difficulté, des modèles simples d'émission de champ électromagnétique par les sous-systèmes sont proposés. En particulier, le développement multipolaire tronqué permet cette modélisation à l'aide d'un nombre réduit de sources élémentaires (dipôle, quadripôle, octopôle...) [WIK85]. La méthode de détermination de ces sources est la suivante : à partir de mesures du champ proche généré par le Dispositif Sous Test (D.S.T.), certaines composantes du développement multipolaire sont identifiées. Le champ est alors connu partout dans l'espace entourant l'appareil, au-delà de la distance de mesure.

Toutefois, la détermination de ces composantes est sensible aux erreurs de mesure. Pour pallier ce problème, les auteurs de [KER03] proposent de multiplier les points de mesures : dans ce travail, le capteur de champ magnétique est une petite antenne boucle, déplacée autour de l'objet étudié afin de mesurer le champ en de nombreux points (environ 200). L'identification des composantes est obtenue en appliquant une technique de type moindre carré. Cependant, la mise en place de ce dispositif reste compliquée et le temps de mesure est important. Dans [ROU06], les mesures sont encore ponctuelles mais cette fois les capteurs sont fixes et leur nombre est optimisé. Cette solution, complètement statique, permet de limiter les incertitudes liées au positionnement.

D'autres méthodes font appel à la mesure du flux induit dans de grandes bobines placées autour du dispositif. Ceci permet, par une intégration spatiale du champ, de réduire l'effet des erreurs de positionnement du capteur [KIL97]. L'approche détaillée dans cet article s'inspire du système d'antennes cadres de la norme CISPR 16-1, dans la bande de fréquence : 9kHz-30MHz, illustré figure 1 [CIS02, BER89, PAS92] qui permet l'identification des 3 composantes du dipôle (une par boucle). L'utilisation de 3 spires identiques et de géométrie simple, facilite la construction du capteur. Néanmoins, pour que cette identification soit précise (cf. section 2.2.3.), il faut que ces antennes boucles soient de grande dimension (diamètre de 2 mètres au minimum). Outre l'encombrement, cela entraîne des complications : loin du D.S.T., le champ est faible et donc difficile à détecter. De plus, son fonctionnement est limité en fréquence. En effet, l'apparition de phénomènes de propagation dans les antennes pose des problèmes de mesure aux environs de $30 \mathrm{MHz}$ [SAV04].

Deux améliorations de ce dispositif sont proposées : ajouter l'identification des composantes du quadripôle et réduire les dimensions du capteur. Ce dernier aspect oblige à trouver de nouveaux contours de détection. Ils doivent présenter des 
propriétés améliorées de filtrage spatial des composantes du développement multipolaire : chaque capteur, constitué de plusieurs bobines, ne doit être sensible qu'à une seule composante parmi toutes les autres. Plus précisément, l'application vise l'identification de l'une des 3 composantes dipolaires du champ, ou de l'une de ses 5 composantes quadripolaires (ordres 1 et 2 du développement), sans être perturbée par les 2 ordres suivants (3 et 4). Si la possibilité de trouver ce jeu de nouveaux capteurs est déjà démontrée [VIN09a], reste à chercher des contours qui permettent de simplifier leurs réalisations. C'est l'objet de cet article. L'ensemble de la démarche (conception et simplification des contours, construction des capteurs et leur validation expérimentale) est regroupé dans un manuscrit de thèse [VIN09b].

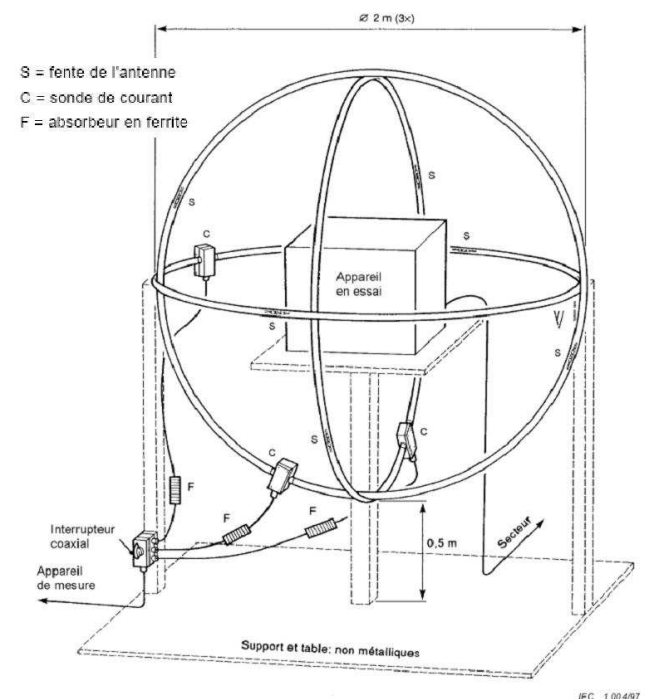

Figure 1. Système de mesure de la norme CISPR 16-1 : 3 spires blindées en courtcircuit et de grand diamètre entourent le D.S.T. Les signaux sont extraits à l'aide de sondes de courant $(C)$ [CISO2]

Dans une première partie est rappelé le développement multipolaire et l'intérêt de son utilisation. Puis la méthode permettant de trouver les contours est détaillée.

\section{Développement multipolaire}

Le développement multipolaire est un développement en série classique des champs électromagnétiques. Dans le cadre de nos mesures en champ proche, le 
recours à l'approximation quasi-statique, pour laquelle il n'y a pas de phénomènes de propagation, permet la simplification du développement [JAC98], [WIK85].

\subsection{Décompositions harmoniques en temps et en espace}

\subsubsection{Décomposition harmonique en temps}

Dans l'air et à l'extérieur d'une sphère (nommée sphère de validité) englobant toutes les sources, le champ magnétique peut être décrit par un potentiel scalaire magnétique $\psi$. La transformée de Fourier de $\psi$ est définie par :

$$
\Psi(r, \theta, \varphi, f)=\int_{-\infty}^{+\infty} \psi(r, \theta, \varphi, t) e^{-j 2 \pi f t} d t
$$

La sphère de validité devant entièrement circonscrire la source, en pratique, son rayon correspond à la moitié de la plus grande dimension de l'objet à caractériser. Ainsi, à l'extérieur de cette sphère et pour chaque fréquence $f, \Psi$ est solution de l'équation de Laplace :

$$
\Delta \Psi(r, \theta, \varphi, f)=0 .
$$

\subsubsection{Décomposition harmonique en espace}

Une solution de l'équation [1] s'écrit sous la forme :

$$
\Psi(r, \theta, \phi, f)=\frac{\mu_{0}}{4 \pi} \sum_{n=1}^{+\infty} \sum_{m=-n}^{+n} A_{n m}(f) \frac{1}{r^{n+1}} S_{n m}(\theta, \phi),
$$

où :

- les $S_{n m}$ sont les harmoniques sphériques réelles, d'ordre $n$ et de degré $m$ décrites dans la sous section 2.3 ,

- le centre de la décomposition est l'origine du système de coordonnées sphériques $(r, \theta, \varphi)$,

- les $A_{n m}$ sont les coefficients de la décomposition, nos inconnues.

L'induction magnétique correspondante est :

$\mathbf{B}(r, \theta, \varphi, f)=-\operatorname{grad}(\Psi(r, \theta, \varphi, f))$,

ou encore : $\mathbf{B}(r, \theta, \varphi, f)=\sum_{n=1}^{+\infty} \sum_{m=-n}^{+n} \mathbf{B}_{n m}$,

avec : $\mathrm{B}_{n m}=-\frac{\mu_{0}}{4 \pi} A_{n m}(f) \operatorname{grad}\left(\frac{1}{r^{n+1}} S_{n m}(\theta, \phi)\right)$. 
Dans la suite, la fréquence étant fixe et donnée, elle est volontairement omise des expressions.

\subsection{Intérêt}

\subsubsection{Hiérarchie des ordres}

Le choix de cette représentation est motivé principalement par le fait que ce développement est hiérarchique: les composantes d'ordres $n$ de l'induction décroissent comme $1 / r^{(n+2)}$. En conséquence, lorsque l'on s'éloigne de la source, le nombre d'ordres nécessaire pour décrire le champ avec une précision donnée décroît.

\subsubsection{Ordre maximum des sources}

De manière générale, une décomposition limitée à l'ordre 2 donne une précision jugée suffisante au-delà de la distance de mesure [KIL97]. Or, ceci correspond aux comportements de la plupart des sources dans les systèmes de l'électronique de puissance. En effet, ces sources correspondent souvent à des boucles de courant. Une étude générique des convertisseurs de puissance [VIN09b] a permis de mettre en évidence la présence majoritaire de sources d'ordre $n=1$ (3 composantes correspondant au dipôle) et d'ordre $n=2$ (5 composantes correspondant au quadripôle). En particulier :

- pour le dipôle : toutes les boucles de courant (formées par les pistes de cuivre ou via les capacités parasites de la structure) sont susceptibles de générer des composantes dipolaires, et ce, dans toutes les directions,

- pour le quadripôle : deux situations bien distinctes sont à l'origine de ce type de composantes. D'une part, toute composante dipolaire décalée par rapport au centre de la décomposition se superpose à des composantes d'ordre supérieur. Ce phénomène est d'autant plus prononcé que la distance au centre est importante et que l'ordre est faible si bien que cela se concrétise par l'apparition majoritaire de ces composantes d'ordre $n=2$. D'autre part, structurellement, la présence systématique d'un plan de masse (semelle métallique plus ou moins plane) occasionne, par l'intermédiaire du théorème des images, des composantes quadripolaires.

Cette étude générique permet finalement de limiter le nombre d'inconnues à identifier à 8 , correspondant aux deux premiers ordres du multipôle équivalent.

\subsubsection{Objectifs}

Ainsi, l'objectif est de déterminer les 8 premières composantes multipolaires magnétiques à partir de mesures réalisées autour des appareils dont nous cherchons à caractériser la perturbation magnétique. Pour cela, une solution possible consiste à s'appuyer sur la décroissance plus rapide des ordres supérieurs avec la distance : suffisamment loin de la source, les capteurs ne sont sensibles qu'au dipôle. La norme 
CISPR 16-1 s'appuie sur cette propriété [PAS92]. Ceci explique la grande dimension des boucles. Pour réduire la taille des capteurs, nous proposons de ne plus compter uniquement sur la décroissance des ordres mais de s'appuyer sur les propriétés géométriques des harmoniques sphériques. Le dessin des bobines doit alors être modifié afin de réaliser un filtrage pour ne mesurer qu'une composante $\mathbf{B}_{n m}$ parmi toutes celles présentes.

\subsection{Les harmoniques sphériques réelles}

Les fonctions harmoniques sphériques réelles d'ordre $n$ et de degré $m$, sont définies par :

$$
S_{n m}(\theta, \phi)=\Theta_{n m}(\theta) \Phi_{m}(\phi),
$$

avec,

$$
\begin{gathered}
\Theta_{n m}(\theta)=\sqrt{\frac{(n-|m|) !}{(n+|m|) !} P_{n|m|}(\cos (\theta)),} \\
\Phi_{m}(\varphi)=\left\{\begin{array}{ccc}
\sqrt{2} \cos (m \varphi) & m>0 \\
1 & \text { if } & m=0 . \\
\sqrt{2} \sin (|m| \varphi) & & m<0
\end{array}\right.
\end{gathered}
$$

Les fonctions $P_{n m}$ sont les fonctions de Legendre associées qui peuvent être définies grâce à la formule de Rodrigues [NED01]. La définition [3] permet de constater que :

$$
S_{n m}(\theta, \varphi+\pi /(2 m))=-S_{n,-m}(\theta, \varphi)
$$

Les fonctions $S_{n m}$ et $S_{n,-m}$ sont donc identiques à une rotation de $\pi /(2 m)$ autour de l'axe $z$ près (les angles du repère de coordonnées sphériques sont rappelés sur la Figure 2a). La détermination des 8 capteurs se limitent donc à la recherche de 5 formes distinctes seulement (2 pour l'ordre 1, 3 pour l'ordre 2). Dans la suite, l'étude porte, pour chaque ordre $n$, uniquement sur les cas où $m \geq 0$. Les fonctions $S_{n m}$ sont représentées sur la figure 2 jusqu'à l'ordre 4 . Les ordres correspondent aux lignes, les degrés, aux colonnes. Sur les deux premières lignes apparaissent les composantes recherchées. Les autres correspondent à celles que l'on va chercher à filtrer. Deux représentations des harmoniques sphériques sont proposées. Figure 2a, les fonctions angulaires sont représentées sur une sphère, en niveaux de gris. Figure $2 \mathrm{~b}$, en plus, l'amplitude du rayon de cette sphère est modulée par la valeur absolue de la fonction harmonique sphérique considérée pour chaque orientation $(\theta, \varphi)$. Ainsi, les lobes apparaissent clairement. Selon les besoins, l'une ou l'autre des représentations est utilisée dans l'exposé. 


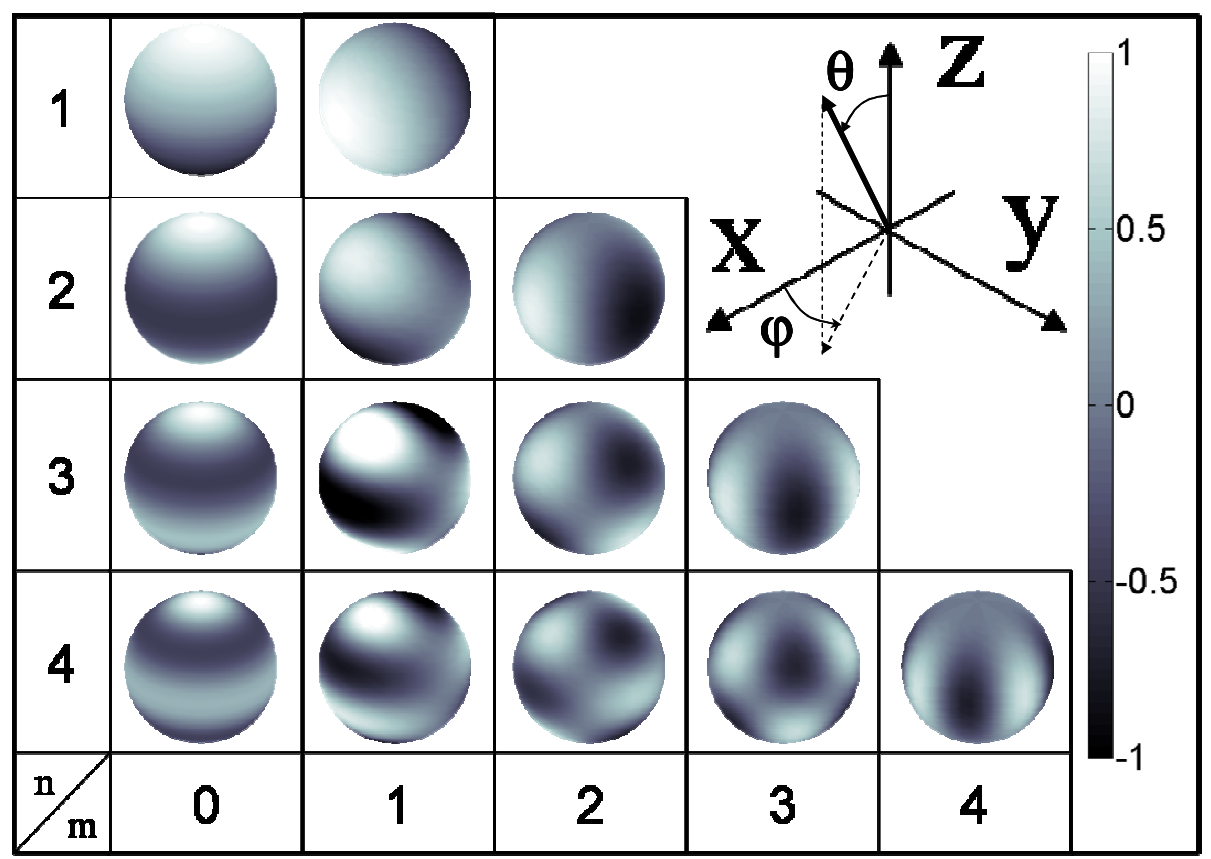

Figure 2a. Représentations des fonctions harmoniques sphériques réelles pour les cas où $n \leq 4$ et $m \geq 0$. Le niveau de gris sur la sphère correspond à la valeur de la fonction harmonique. 


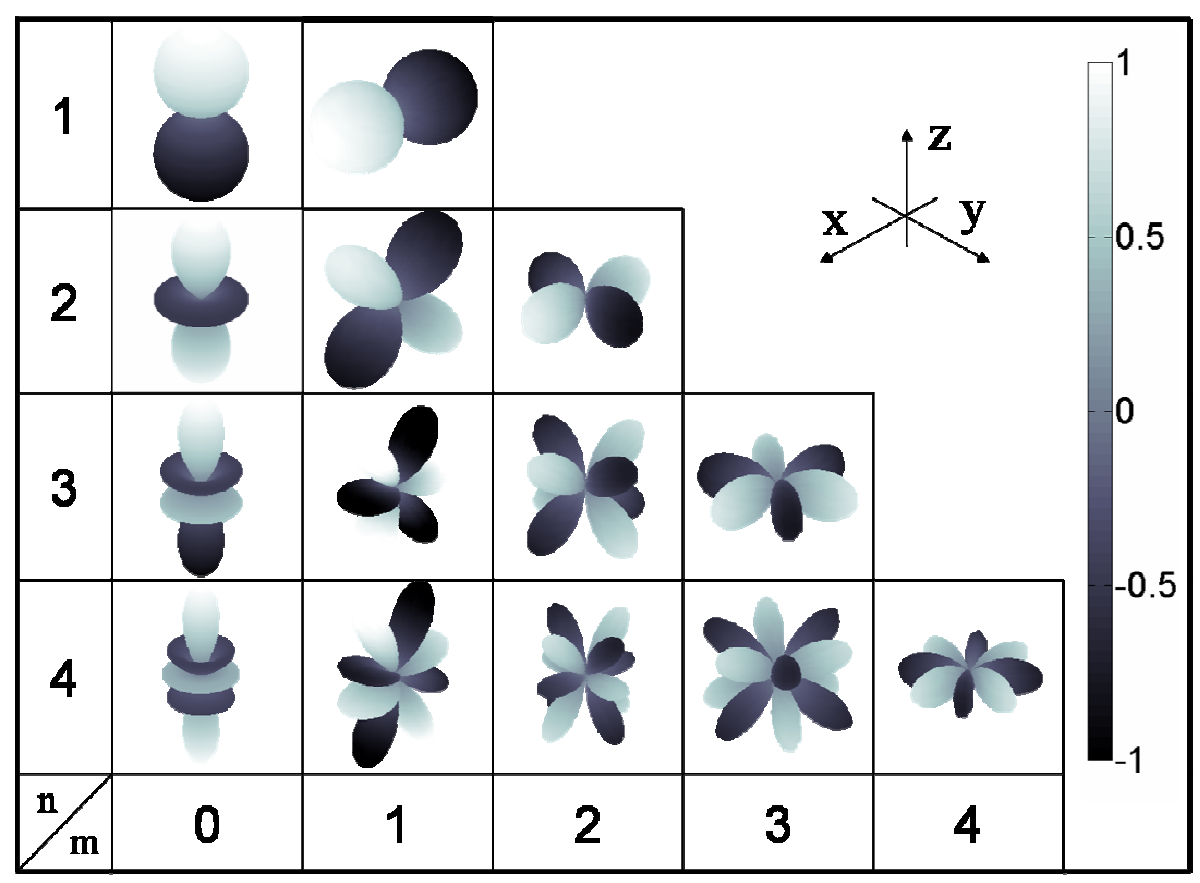

Figure 2b. Représentations des fonctions harmoniques sphériques réelles pour les cas où $n \leq 4$ et $m \geq 0$. Le niveau de gris sur la sphère correspond à la valeur de la fonction harmonique. Le rayon de la sphère est également modulé par la valeur absolue de la fonction harmonique.

\section{Création des capteurs de flux}

\subsection{Préliminaires}

Chaque capteur, spécifique à une composante donnée du développement multipolaire, est constitué de plusieurs spires. Elles sont dessinées sur la sphère de mesure de rayon $r_{0}$ notée $S_{M}$ et englobant la sphère de validité. Un exemple de contour est donné sur la figure 3 . Le capteur qui identifie la composante $A_{n m}$ (voir la relation [2]) est noté $C^{n m}$. Si la sphère de mesure doit englober celle de validité, le choix définitif de la valeur de $r_{0}$ reste directement relié à l'exigence sur la précision de l'identification [VIN09b]. 

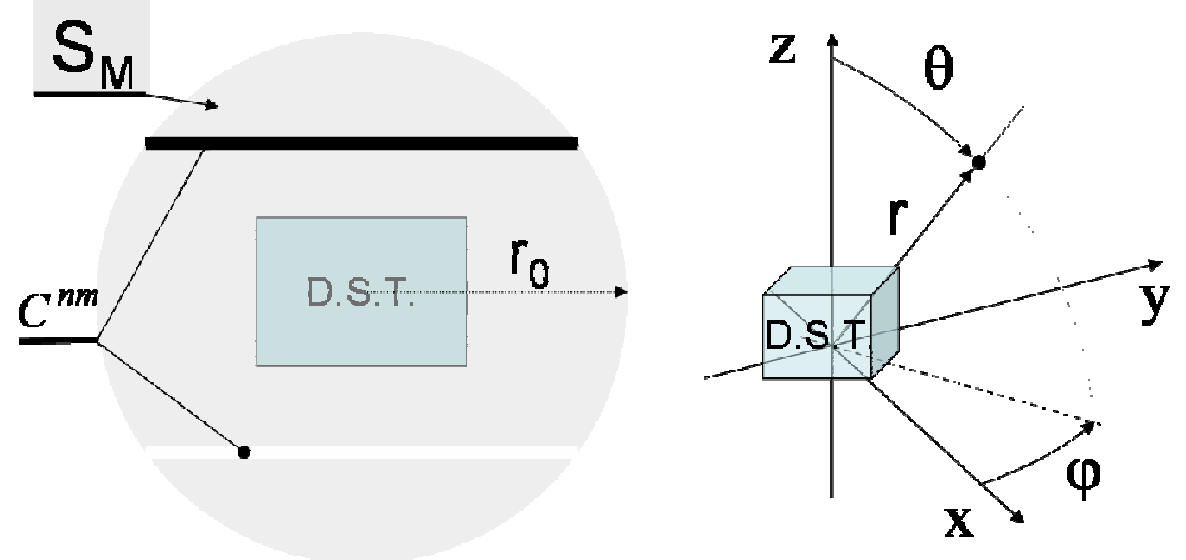

Figure 3. A gauche, en vue de face, le capteur $C^{n m}$ d'une composante $A_{n m}$, constitué de bobines (en noir et blanc), dessinées sur la sphère $S_{M}$, de rayon $r_{0}$ et centrée sur le D.S.T. A droite, la définition du repère de coordonnées utilisé

Le principe consiste à mesurer des flux d'induction à travers les spires et de faire des sommes ou des différences de ces flux. Ceci est symbolisé sur les représentations, figure 4 , en dessinant en noir une spire orientée de telle sorte que le flux embrassé soit compté positivement, en blanc de telle sorte que le flux soit compté négativement.

Ce jeu de spires sur la sphère $S_{M}$ peut alors être représenté mathématiquement par une « fonction capteur » $C$ définie par :

- $C(\theta, \varphi)=1$ sur une portion de $S_{M}$ délimitée par une spire noire,

- $C(\theta, \varphi)=-1$ sur une portion de $S_{M}$ délimitée par une spire blanche,

- $C(\theta, \varphi)=0$ sur les portions de $S_{M}$ restantes.

La quantité mesurée correspond alors à :

$$
\text { Mesure }=\int_{S_{M}} C(\theta, \varphi) \mathbf{B}\left(r_{0}, \theta, \varphi\right) \cdot \mathbf{n} d s=\int_{S_{M}} C(\theta, \varphi) B_{r}\left(r_{0}, \theta, \varphi\right) d s
$$

où $\boldsymbol{n}$ est la normale unitaire sortante de $S_{M}$ et $B_{r}\left(r_{0}, \theta, \varphi\right)$, la composante radiale du champ sur $S_{M}$, définie par :

$$
B_{r}\left(r_{0}, \theta, \varphi\right)=\frac{\mu_{0}}{4 \pi} \sum_{n=1}^{+\infty} \sum_{m=-n}^{+n} \frac{(n+1)}{r_{0}^{n+2}} A_{n m} S_{n m}(\theta, \varphi)
$$

Par ailleurs, de façon analogue à la décomposition en série de Fourier des signaux temporels périodiques, la fonction capteur $C$ admet un développement en harmoniques sphériques réelles : 


$$
C(\theta, \varphi)=\sum_{n=1}^{+\infty} \sum_{m=-n}^{+n} C_{n m} S_{n m}(\theta, \varphi)
$$

où les coefficients $C_{n m}$ de la décomposition en harmoniques sphériques réelles sont définis par :

$$
C_{n m}=\frac{\int_{S_{M}} C S_{n m} d s}{\int_{S_{M}}\left|S_{n m}\right|^{2} d s}=\frac{(2 n+1)}{4 \pi r_{0}^{2}} \int_{S_{M}} C S_{n m} d s .
$$

En conséquence, la mesure correspond à :

$$
\text { Mesure }=\frac{\mu_{0}}{4 \pi} \sum_{n=1}^{+\infty} \frac{(n+1)}{r_{0}^{n+2}} \sum_{m=-n}^{+n} A_{n m}\left(C_{n m} \int_{S_{M}}\left|S_{n m}\right|^{2} d s\right) \text {. }
$$

Idéalement, l'identification d'une composante $A_{n m}$ particulière impose :

$$
C_{n m} \neq 0 \text { et } C_{p q}=0, \forall(p, q) \neq(n, m) \text {. }
$$

D'après [6], ceci implique que $C$ soit proportionnelle à $S_{n m}$. Or cela n'est pas possible compte tenu de la définition donnée à la fonction $C$, qui peut prendre uniquement les valeurs $-1,0$ ou 1 .

Par contre, nous avons montré que pour les composantes $A_{10}$ et $A_{20}$, figure 4 , les coefficients des capteurs $C^{10}$ et $C^{20}$ vérifient la condition [VIN09a] :

$$
\left\{\begin{array}{l}
C_{p q}^{i j} \neq 0 \text { pour }\left\{\begin{array}{l}
p=i \\
q=j \\
C_{p q}^{i j}=0 \text { pour }\left\{\begin{array}{l}
p \neq i \\
q \neq j
\end{array}\right.
\end{array} \quad \forall(p \in[1 ; 4] ; q \in[-p ; p])\right.
\end{array}\right.
$$

Autrement dit, il existe des solutions pour mesurer les composantes $A_{10}$ et $A_{20}$ en filtrant toutes les composantes jusqu'à l'ordre 5. Les relations liant les composantes et les flux mesurés sont :

$$
A_{10}=\frac{10^{8} r_{0}}{32 \pi}\left(\text { flux }_{1}-\text { flux }_{2}\right)
$$

où les flux sont ceux mesurés à travers les bobines de la fonction $C^{10}$ sur la figure 4 , et :

$$
A_{20}=\frac{6125 \times 10^{4} r_{0}^{2}}{3 \pi \sqrt{21}}\left(\text { flux }_{1}+\text { flux }_{2}\right)
$$

où les flux sont ceux mesurés à travers les bobines de la fonction $C^{20}$ sur la figure 4 . Les calculs de ces expressions sont détaillés en annexe. 
On souhaite généraliser la propriété [9] pour définir des capteurs pour toutes les composantes des ordres 1 et 2 . Ceci consiste à isoler une composante parmi 24 possibles (toutes les composantes des ordres 1 à 4). Dans la suite, en accord avec [9], l'effet perturbateur des ordres 5 et plus dans les capteurs est négligé.

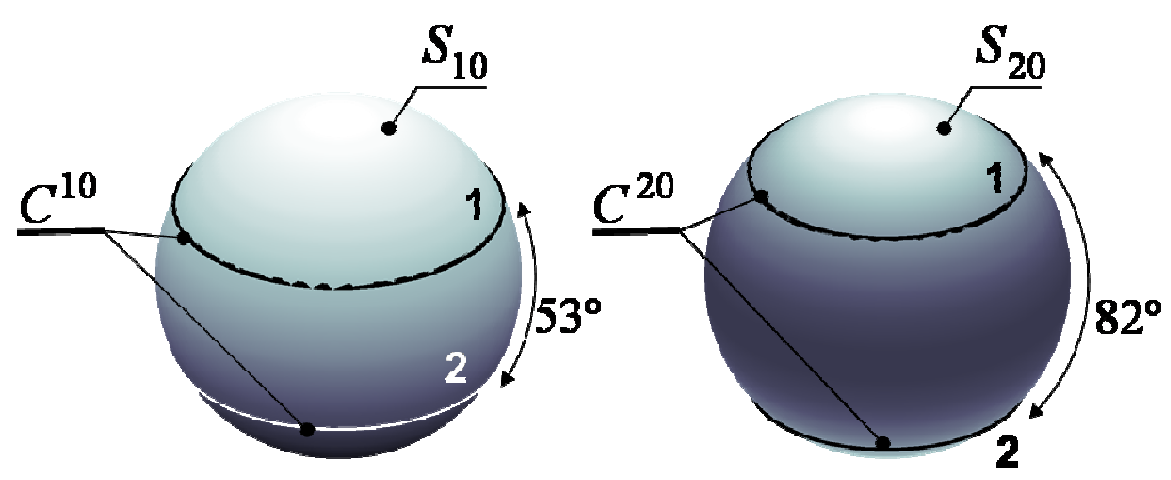

Figure 4. Capteurs de détection $C^{10}$ et $C^{20}$ et leurs 2 composantes associées. Les positions des bobines sont symétriques par rapport à l'équateur

\subsection{Construction du jeu de capteurs}

La figure 5 permet d'illustrer l'idée sous-jacente de notre approche. En effet, les différentes composantes d'un même ordre peuvent être décrites par des formes similaires. Par exemple, la fonction $S_{11}$ est obtenue par rotation de $S_{10}$ d'un angle de $\pi / 2$ autour de l'axe $O y$. Un même constat peut être fait entre $S_{21}$ et $S_{22}$ : la transformation dans ce cas se compose d'une première rotation de $\pi / 4$ autour de $O y$ puis d'une seconde de $\pi / 2$ autour de $O x$. Dans la suite, il est démontré qu'une seule forme par ordre est nécessaire pour définir toutes les autres à l'aide de rotations. L'idée est que l'on peut généraliser cette propriété pour concevoir les capteurs, en partant des deux présentés figure 4. 


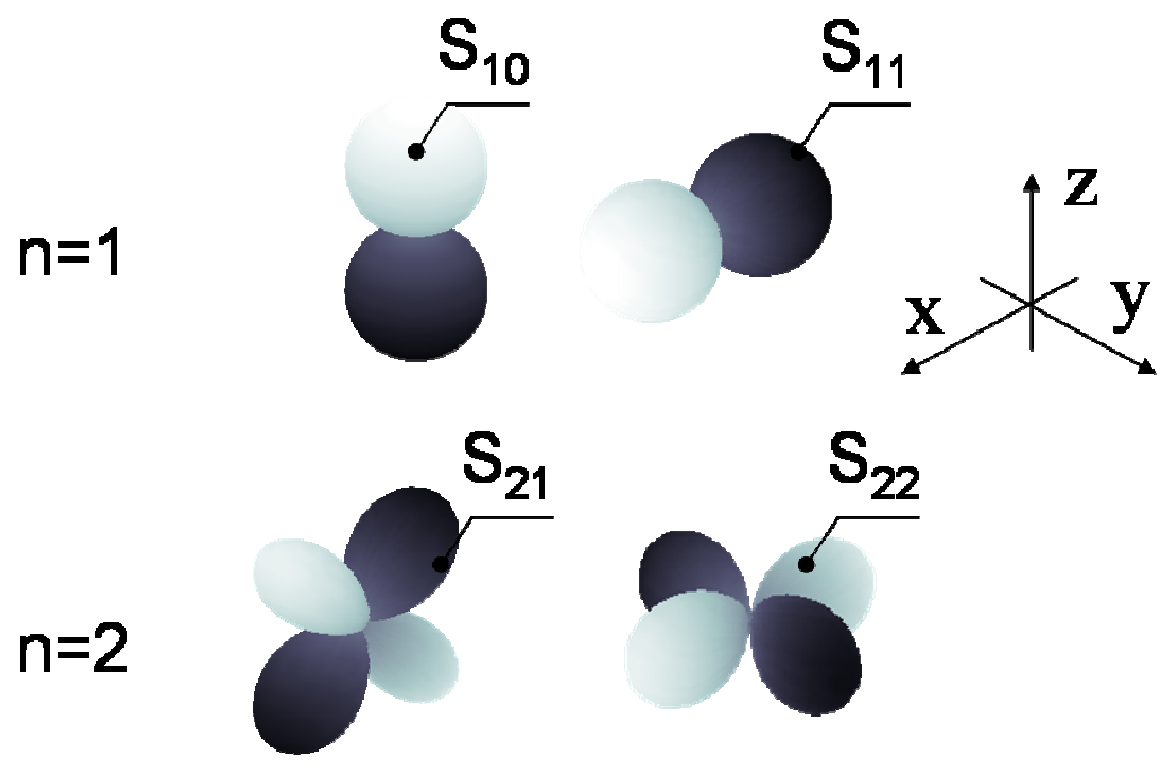

Figure 5. Similitudes entre les formes des harmoniques sphériques

\subsubsection{Conventions prises pour la description d'une rotation}

La transformation par rotation s'appuie sur les angles d'Euler, dans l'ordre présenté sur la figure 6 . Une rotation quelconque se décompose alors en trois rotations élémentaires : une première rotation d'angle $\alpha$ autour de l'axe $O z$ suivie d'une deuxième d'un angle $\beta$ autour de l'axe $O y$ ' et une dernière d'un angle $\gamma$ autour de l'axe $O z^{\prime}$. Dans la suite, $R(\alpha, \beta, \gamma)$ désigne cette rotation et $(O, \tilde{x}, \tilde{y}, \tilde{z})$ le repère tourné après avoir subit la rotation $R(\alpha, \beta, \gamma)$.
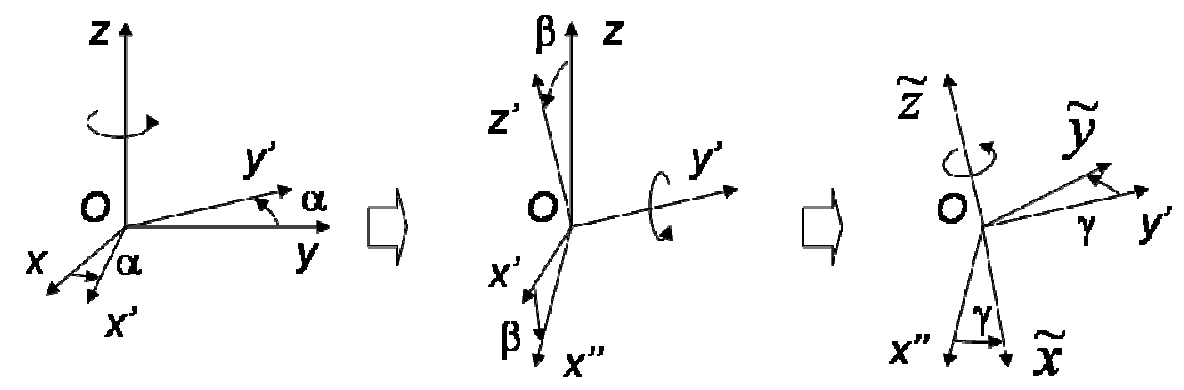

Figure 6. Description d'une rotation à l'aide des angles d'Euler $(\alpha, \beta, \gamma)$ 


\subsubsection{Rotation des harmoniques sphériques}

On note $S_{n m}$ les harmoniques sphériques réelles non normées dans le repère de référence $(O, x, y, z)$ et $\tilde{S}_{n m}$ celles dans le repère $(O, \tilde{x}, \tilde{y}, \tilde{z})$.

Les fonctions $S_{n m}$, et $\tilde{S}_{n m}, m \in[-n ; n]$ forment des bases orthogonales de l'espace de fonctions d'ordre $n$ d'un développement en harmoniques sphériques. Il existe donc une matrice de passage d'une base à l'autre, soit une matrice $R^{(n)}$ permettant d'écrire :

$$
\tilde{S}_{n m}=\sum_{m^{\prime}=-n}^{n} R_{m^{\prime} m}^{(n)} S_{n m^{\prime}}, \forall m \in[-n ; n]
$$

En particulier, on s'intéresse aux vecteurs de coordonnées de $\tilde{S}_{10}$ et $\tilde{S}_{20}$ dans les bases $S_{n m}, m \in[-n ; n], n=1$ ou 2 , donnés par :

$$
R^{(1)}\left[\begin{array}{l}
0 \\
1 \\
0
\end{array}\right]=\left[\begin{array}{c}
\sin (\alpha) \sin (\beta) \\
\cos (\beta) \\
\cos (\alpha) \sin (\beta)
\end{array}\right]
$$$$
\text { et } \quad R^{(2)}\left[\begin{array}{l}
0 \\
0 \\
1 \\
0 \\
0
\end{array}\right]=\left[\begin{array}{c}
\sqrt{3} / 2 \sin (2 \alpha) \sin ^{2}(\beta) \\
\sqrt{3} / 2 \sin (\alpha) \sin (2 \beta) \\
1 / 4(1+3 \cos (2 \beta) \\
\sqrt{3} / 2 \cos (\alpha) \sin (2 \beta) \\
\sqrt{3} / 2 \cos (2 \alpha) \sin ^{2}(\beta)
\end{array}\right]
$$

Les matrices $R^{(1)}$ et $R^{(2)}$ nécessaires sont données dans [GRE03].

Si $S_{10}^{\alpha, \beta}$ désigne l'harmonique $\tilde{S}_{10}$ dans le repère tourné suivant la rotation $R(\alpha, \beta, 0)$, on vérifie alors que, pour $n=1$ :

$$
S_{10}^{0, \pi / 2}=S_{11} \text { et } S_{10}^{\pi / 2, \pi / 2}=S_{1,-1} .
$$

Ceci confirme les observations faites figure 5: toutes les composantes de l'ordre 1 peuvent être générées par rotation de $S_{10}$. La composante $S_{11}$ est obtenue après une rotation de $\pi / 2$ autour de $O y$ et $S_{1,-1}$ après une rotation de $\pi / 2$ autour de $O x$.

Concernant l'ordre 2, à l'aide des mêmes notations, les relations suivantes sont obtenues :

$$
\begin{aligned}
& S_{20}^{0, \pi / 4}=\frac{1}{4} S_{20}+\frac{\sqrt{3}}{2} S_{21}+\frac{\sqrt{3}}{4} S_{22}, \\
& S_{20}^{0,-\pi / 4}=\frac{1}{4} S_{20}-\frac{\sqrt{3}}{2} S_{21}+\frac{\sqrt{3}}{4} S_{22}, \\
& S_{20}^{0, \pi / 2}=-\frac{1}{2} S_{20}+\frac{\sqrt{3}}{2} S_{22},
\end{aligned}
$$




$$
S_{20}^{\pi / 2, \pi / 2}=-\frac{1}{2} S_{20}-\frac{\sqrt{3}}{2} S_{22} .
$$

Par combinaison linéaire, s'en suit :

$$
\begin{aligned}
& S_{21}=\frac{1}{\sqrt{3}}\left(S_{20}^{0, \pi / 4}-S_{20}^{0,-\pi / 4}\right) \\
& S_{22}=\frac{1}{\sqrt{3}}\left(S_{20}^{0, \pi / 2}-S_{20}^{\pi / 2, \pi / 2}\right)
\end{aligned}
$$

Comme pour l'ordre 1, les relations [12] et [13] montrent que toutes les composantes de l'ordre 2 peuvent être générées par rotation de $S_{20}$. En effet, d'après la relation [4], la construction de $S_{2,-1}\left(\right.$ de $S_{2,-2}$ ) est déduite par rotation de $\pi / 2$ de $S_{21}$ (de $\pi / 4$ de $S_{22}$ ) autour de l'axe $O z$.

\subsubsection{Effet des rotations sur la mesure}

D'après [6], après une rotation quelconque du capteur, la fonction $C$ dans le repère tourné conserve les mêmes coefficients $C_{n m}$ que dans le repère initial :

$$
C(\theta, \phi)=\sum_{n \geq 0} \sum_{m=-n}^{+n} C_{n m^{\prime}} \tilde{S}_{n m}(\theta, \phi)=\sum_{n \geq 0} \sum_{m=-n}^{+n} C_{n m} \sum_{m^{\prime}=-n}^{n} R_{m{ }^{\prime} m}^{(n)} S_{n m^{\prime}}(\theta, \phi)
$$

D'après [8], le flux mesuré grâce à $C$ s'écrit alors :

$$
\text { Mesure }=\frac{\mu_{0}}{4 \pi} \sum_{n \geq 0} \frac{(n+1)}{r_{0}^{n+2}} \sum_{m=-n}^{+n} A_{n m}\left(\int_{S_{M}}\left|S_{n m}\right|^{2}\right) \sum_{m^{\prime}=-n}^{+n} R_{m m^{\prime}}^{(n)} C_{n m^{\prime}}(\theta, \phi)
$$

En conséquence, si le capteur tourne, d'autres composantes de l'induction peuvent influer sur la mesure mais elles sont toutes du même ordre $n$. Or, le jeu de capteurs présentés figure 4 permet de repousser les perturbations jusqu'à l'ordre 5 . Cette caractéristique est donc maintenue quelles que soient les rotations effectuées sur ces capteurs.

\subsubsection{Exemple de réalisation du capteur $C^{21}$}

Le processus de création du capteur $C^{21}$ est donné sur la figure 7. Le point de départ correspond à la composante $S_{20}$ et à son capteur $C^{20}$, voir figure 4. [12] précise les deux rotations à effectuer sur cet ensemble pour obtenir la composante $S_{21}$ et son capteur associé $C^{21}$. Enfin, la relation liant la mesure et la composante $A_{21}$ est démontrée en annexe et donnée par :

$$
A_{21}=\frac{6125 \cdot 10^{4} \cdot r_{0}^{2}}{3 \pi \sqrt{21}}\left(\frac{1}{\sqrt{3}}\right)\left(\text { flux }_{1}+f l u x_{2}-f l u x_{3}-f l u x_{4}\right),
$$


où les flux sont ceux mesurés à travers les bobines de la fonction $C^{21}$ sur la figure 7.
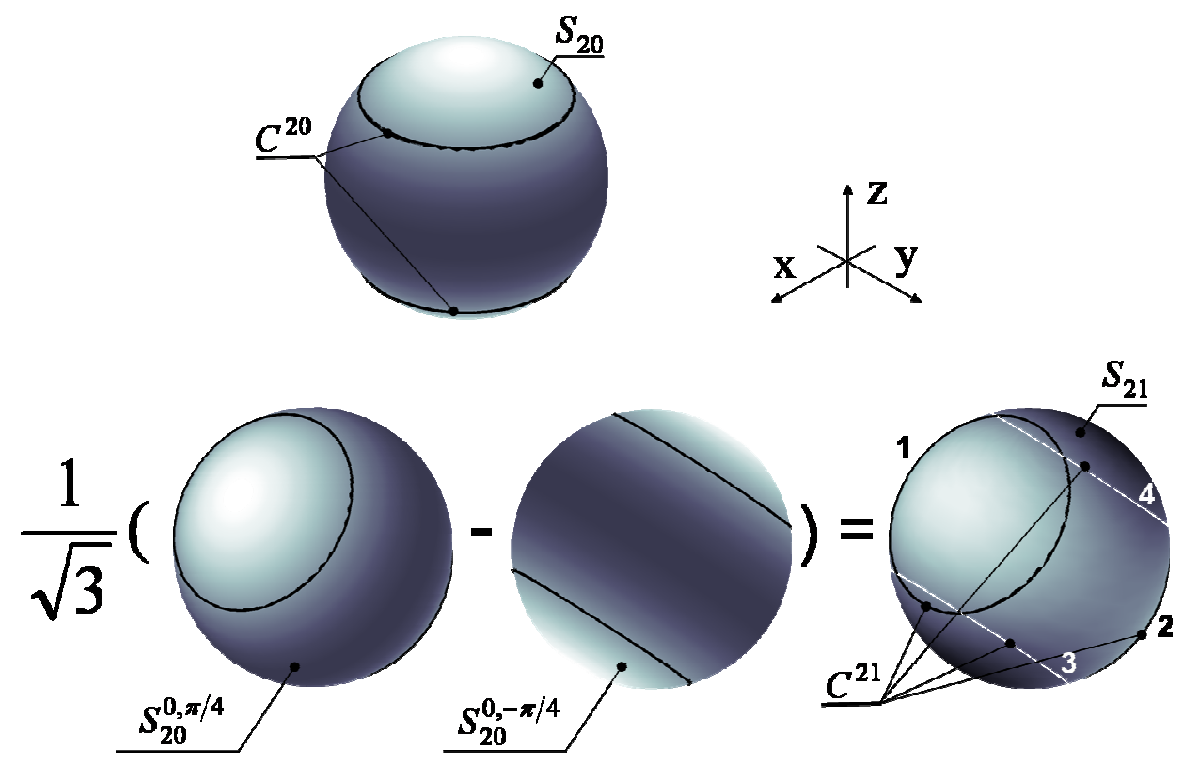

Figure 7. Détail de la création du capteur $C^{21}$

\subsubsection{Présentation de l'ensemble des contours}

Toutes les fonctions ont été créées à partir de la méthode décrite en sous section 3.2.4. En effet, la détermination de $C^{22}$ est analogue à celle de $C^{21}$ mais en s'appuyant sur la relation [13]. Enfin, les contours des fonctions avec $m<0$ sont obtenus par simple rotation de $\pi /(2 m)$ autour de l'axe $O z$ de la fonction à $m>0$ correspondante. Concernant l'ordre 1 , les relations sont immédiates et données par [10].

Les résultats sont regroupés sur la figure 8. Par rapport à la version précédente des capteurs [VIN09a], toutes les bobines de détection sont maintenant planes. Les trois capteurs pour $n=1$ sont identiques à une rotation près. Pour $n=2$, les 4 capteurs à double paire de spires sont identiques à une rotation près ; les spires du capteur $\mathrm{C}^{20}$ sont en plus géométriquement identiques à chacune des paires de spires des capteurs à double paire. 


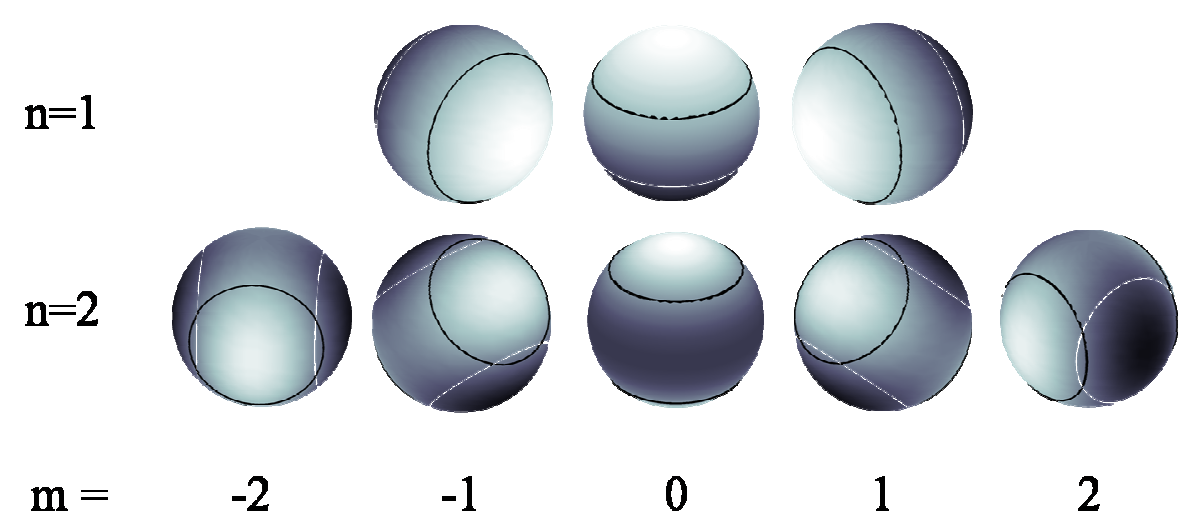

Figure 8. Jeu des 8 nouveaux capteurs

\section{Conclusion}

Nous avons montré qu'il est possible d'améliorer le dispositif de mesure des composantes dipolaires du champ magnétique proposé par la norme. A précision équivalente sur l'identification des composantes [VIN09b], cette version est de dimension plus petite. Mis à part le gain sur l'encombrement, cette amélioration doit permettre d'explorer une bande de fréquence plus large, du fait de l'apparition repoussée des effets de résonance, liées aux phénomènes de propagation sur les bobines. Concrètement, une réduction d'un facteur trois sur le diamètre doit permettre d'étendre la bande passante du capteur. Ainsi, la limite actuelle de 30 $\mathrm{MHz}$ pourrait être repoussée jusqu'à environ $100 \mathrm{MHz}$.

De plus, démonstration est faite qu'il est également possible de créer des systèmes de mesure des composantes quadripolaires. Ainsi, la connaissance du champ généré est possible même dans une zone proche de la source. En outre, cela permet effectivement de caractériser des sources de type quadripolaire présentes dans les dispositifs, ce dont ne rend pas compte le capteur normalisé.

Jusqu'à présent, les identifications à l'aide de ces jeux de capteurs ont été réalisées sous forme d'expériences numériques. Les performances obtenues confirment les attentes. Fort des simplifications apportées avec cette version de capteur, la réalisation et la validation expérimentale sont en cours [VIN09b]. 


\section{Bibliographie}

[AIM09] Aimé J., « Rayonnement des convertisseurs statiques. Application à la variation de vitesse, » 2009, Thèse de doctorat de l'Université Joseph Fourier de Grenoble.

[BER89] Bergervoet J.R. et Van Veen H., «A large-loop antenna for magnetic field measurements », Actes de Zurich International Symposium on EMC, 1989, p.:29-34.

[BLA97] Blanco M.A., Florez M., Bermejo M., «Evaluation of the rotation matrices in the basis of real spherical harmonics », Journal of Molecular structure : THEOCHEM, 1997, 419(1-3) : 19,27.

[CIS02] Norme CISPR 16-1, annexe P, 2002, p. 230-237 et 396-409.

[GRE03] Green R., «Spherical harmonic lighting: the gritty details. », Actes de the Game Developers Conference, 2003, p. 1-47.

[JAC98] Jackson J.D., Classical Electrodynamics, $3^{\text {ième }}$ ed., 1998, pp.407-456.

[KER03] Keradec J.P., Lorange J., Schanen J.-L., Cogitore B., Creuzet T., Brun C., « Nanoteslameter for characterization of the fields emitted and received by wound electronic components », Actes de I.M.T.C., 2003, vol.1, p. 124-129.

[KIL97] Kildishev A.V., Volokhov S.A., Saltykov J.D., «Measurement of the spacecraft main magnetic parameters », Actes de IEEE Autotescon, 1997, p. 669-675.

[NED01] Nedelec J.C., Acoustic and electromagnetic equations: integral representations for harmonic problems, Springer, 2001.

[PAS92] Pasmooij W. A., «A 'Helmholtz' large loop antenna system for improved magnetic field measurements », Actes de conference on E.M.C., 1992, p. 143-148.

[ROU06] Rouve L.-L., Schmerber L., Chadebec O., Foggia A., «Optimal magnetic sensor location for spherical harmonic identification applied to radiated electrical devices », IEEE Trans. on Mag., 42-4, 2006, p. 1167-1170.

[SAV04] Savi M., Gireli T.Z., Tirich F.A., Sartori C.A.F., «Developing a Van Veen \& Bergervoet Antenna », Actes de conference on E.M.C., 2004, p. 717-720.

[VIN09a] Vincent B., Chadebec O., Schanen J.-L., Sartori C.A.F., Krähenbühl L., Perrussel R., Berger K., «New robust coil sensors for near field characterization », Journal of Microwaves, Optoelectronics and Electromagnetic Applications, 2009, Vol. 8 (1), 64S$77 \mathrm{~S}$.

[VIN09b] Vincent B., «Identification de sources électromagnétiques multipolaires équivalentes par filtrage spatial : Application à la CEM rayonnée pour les convertisseurs d'électronique de puissance », 2009, Thèse de doctorat de l'Institut National Polytechnique de Grenoble.

[WIK85] Wikswo J.P., Swinney K.R., "Scalar multipole expansions and their dipole equivalents », J. Appl. Phys., 1985, p. 4301-4308. 


\section{Annexe}

\subsection{Expressions des composantes $A_{10}$ et $A_{20}$ en fonction des flux mesurés}

L'équation [8] permet d'exprimer le lien entre les composantes et les flux mesurés à travers les contours. Pour un capteur $C^{n m}$ :

$$
\begin{aligned}
\text { Mesure }_{n m}= & \frac{\mu_{0}}{4 \pi} \sum_{p=1}^{4} \frac{(p+1)}{r_{0}^{p+2}} \sum_{q=-p}^{+p} A_{p q}\left(C_{p q}^{n m} \int_{S_{M}}\left|S_{p q}\right|^{2} d s\right) \\
& +\frac{\mu_{0}}{4 \pi} \sum_{p=5}^{+\infty} \frac{(p+1)}{r_{0}^{p+2}} \sum_{q=-p}^{+p} A_{p q}\left(C_{p q}^{n m} \int_{S_{M}}\left|S_{p q}\right|^{2} d s\right) .
\end{aligned}
$$

En accord avec nos hypothèses, l'effet du second groupe de termes sur la mesure est négligé. En utilisant la condition [9], il vient :

$$
\begin{aligned}
\text { Mesure }_{n m} & =\frac{\mu_{0}}{4 \pi}\left(\frac{(n+1)}{r_{0}^{n+2}}\right) A_{n m}\left(\frac{4 \pi r_{0}^{2}}{(2 n+1)} C_{n m}^{n m}\right) \\
& =4 \pi 10^{-7}\left(\frac{n+1}{2 n+1}\right) \frac{C_{n m}^{n m} A_{n m}}{r_{0}^{n}} .
\end{aligned}
$$

Pour $C^{10}$, on a en conséquence :

$$
\text { Mesure }_{10}=\frac{8 \pi 10^{-7}}{3 r_{0}} A_{10} C_{10}^{10}
$$

avec, d'après [7] et figure $4: C_{10}^{10}=\left(\frac{3}{4 \pi r_{0}^{2}}\right) r_{0}^{2} \int_{0}^{2 \pi} d \varphi \int_{0}^{\pi} C^{10}(\theta, \varphi) \cdot S_{10} \sin \theta d \theta=\frac{6}{5}$,

d'où : $A_{10}=\frac{10^{8} r_{0}}{32 \pi}$ Mesure $_{10}=\frac{10^{8} r_{0}}{32 \pi}\left(\right.$ flux $_{1}-$ flux $\left._{2}\right)$,

où les flux sont ceux mesurés à travers les bobines de la fonction $C^{10}$ sur la figure 4 .

Pour $C^{20}$, on a :

$$
\text { Mesure }_{20}=\frac{12 \pi 10^{-7}}{5 r_{0}^{2}} A_{20} C_{20}^{20},
$$

avec, d'après [7] : $C_{20}^{20}=\left(\frac{5}{4 \pi r_{0}^{2}}\right) r_{0}^{2} \int_{0}^{2 \pi} d \varphi \int_{0}^{\pi} C^{20}(\theta, \varphi) \cdot S_{20} \sin \theta d \theta=\frac{10 \sqrt{21}}{49}$, 
d'où : $A_{20}=\frac{6125 \times 10^{4} r_{0}^{2}}{3 \pi \sqrt{21}}$ Mesure $_{20}=\frac{6125 \times 10^{4} r_{0}^{2}}{3 \pi \sqrt{21}}\left(\right.$ flux $_{1}+$ flux $\left._{2}\right)$,

où les flux sont ceux mesurés à travers les bobines de la fonction $C^{20}$ sur la figure 4 .

\subsection{Expressions de la composante $A_{21}$ en fonction des flux mesurés par le capteur $C^{21}$}

D'après la figure $7, \operatorname{si}\left(C^{n m}\right)^{\alpha, \beta}$ désigne le capteur $C^{n m}$ dans le repère tourné, on a :

$$
C^{21}=\left(C^{20}\right)^{0, \pi / 4}-\left(C^{20}\right)^{0,-\pi / 4}
$$

D'après les conditions [9], ceci équivaut à :

$$
\begin{aligned}
C^{21} & =C_{20}^{20} S^{0, \pi / 4}-C_{20}^{20} S^{0,-\pi / 4}+(\text { termes d'ordre } \geq 5) \\
& =\sqrt{3} C_{20}^{20} S_{21}+(\text { termes d'ordre } \geq 5), \text { d'après [12] }
\end{aligned}
$$

On a donc, d'une part :

$$
\text { Mesure }_{21}=\text { flux }_{1}+\text { flux }_{2}-\text { flux }_{3}-\text { flux }_{4} \text {, }
$$

où les flux correspondent à ceux indiqués sur la figure 7 pour $C^{21}$ et, d'autre part, d'après [8] et l'annexe 6.1. :

$$
\begin{aligned}
\text { Mesure }_{21} & =\int_{S_{M}} C^{21} B_{r} d s=\sqrt{3} C_{20}^{20} \int_{S_{M}} S_{21} B_{r} d s, \text { on néglige les termes d'ordre } \geq 5, \\
& =\sqrt{3} C_{20}^{20} \frac{\mu_{0}}{4 \pi} \frac{(2+1)}{r_{0}^{2+2}} A_{21} \int_{S_{M}}\left|S_{21}\right|^{2} d s .
\end{aligned}
$$

Finalement :

$$
\begin{aligned}
A_{21} & =\frac{4 \pi}{\mu_{0}} \frac{r_{0}^{4}}{3} \frac{1}{C_{20}^{20}}\left(\frac{1}{\sqrt{3}}\right)\left(\text { flux }_{1}+f l u x_{2}-f l u x_{3}-f l u x_{4}\right) \\
& =\frac{6125 \times 10^{4} r_{0}^{2}}{3 \pi \sqrt{21}}\left(\frac{1}{\sqrt{3}}\right)\left(\text { flux }_{1}+f l u x_{2}-f l u x_{3}-f l u x_{4}\right) .
\end{aligned}
$$

